\title{
ON THE KERNELS OF REPRESENTATIONS OF FINITE GROUPS
}

\author{
by SHIGEO KOSHITANI
}

(Received 1 November, 1979)

Let $G$ be a finite group and $p$ a prime number. About five years ago I. M. Isaacs and S. D. Smith [5] gave several character-theoretic characterizations of finite $p$-solvable groups with $p$-length 1 . Indeed, they proved that if $P$ is a Sylow $p$-subgroup of $G$ then the next four conditions (1)-(4) are equivalent:

(1) $G$ is $p$-solvable of $p$-length 1 .

(2) Every irreducible complex representation in the principal p-block of $G$ restricts irreducibly to $N_{G}(P)$.

(3) Every irreducible complex representation of degree prime to $p$ in the principal p-block of $G$ restricts irreducibly to $N_{G}(P)$.

(4) Every irreducible modular representation in the principal p-block of $G$ restricts irreducibly to $N_{G}(P)$.

The purpose of the present paper is to generalize the above result. It can be stated as follows: if $B$ is an arbitrary p-block of $G$ with defect group $D$, then the following four conditions (1)-(4) are equivalent:

(1) $D$ is contained in the intersection of the kernels of all irreducible modular representations in $B$.

(2) Every irreducible complex representation in $B$ restricts irreducibly to $N_{G}(D)$.

(3) Every irreducible complex representation in $B$ whose character has height zero restricts irreducibly to $N_{G}(D)$.

(4) Every irreducible modular representation in $B$ restricts irreducibly to $N_{G}(D)$.

Since $O_{p^{\prime}, p}(G)$ is the intersection of the kernels of all irreducible modular representations in the principal p-block of $G$, our result is a generalization of the result of Isaacs and Smith.

Throughout this paper we use the following notation. For an integer $n$ we write $\nu_{\mathrm{p}}(n)=r$ if $p^{r} \mid n$ and $p^{r+1} \backslash n$. We write $\operatorname{Irr}(G)$ (respectively $\operatorname{IBr}(G)$ ) for the set of all irreducible complex (respectively Brauer) characters of $G$. For a p-block $B$ of $G$ let us denote by $\operatorname{Irr}(B)$ (respectively $\operatorname{IBr}(B)$ ) the set of all elements of $\operatorname{Irr}(G)$ (respectively $\operatorname{IBr}(G)$ ) which belong to $B$, by $k(B)$ the number of elements of $\operatorname{Irr}(B)$, and by $k_{0}(B)$ the number of elements of $\operatorname{Irr}(B)$ with height zero. When $\chi \in \operatorname{Irr}(G)($ respectively $\phi \in \operatorname{IBr}(G))$, let $\operatorname{Ker} \chi$ (respectively $\operatorname{Ker} \phi$ ) be the kernel of the irreducible complex (respectively modular) representation which corresponds to $\chi$ (respectively $\phi$ ). Following [1, pp. 494-495] let $N_{B}=\bigcap\{\operatorname{Ker} \chi \mid \chi \in \operatorname{Irr}(B)\}$ and $N_{B}^{*}=\bigcap\{\operatorname{Ker} \phi \mid \phi \in \operatorname{IBr}(B)\}$ for a $p$-block $B$ of $G$. We write $B_{0}(G)$ for the principal p-block of $G$. When $H$ is a subgroup of $G$ and $b$ is a $p$-block of $H$, we use the notation $b^{G}$ in the sense of $[3, \S 57]$ for the case where $b^{G}$ is defined. When $H$ is a subgroup of $G$, for a character $\psi$ of $G$ and a character $\tilde{\psi}$ of $H,\left.\psi\right|_{H}$ and $\tilde{\psi}^{G}$ denote the restriction of $\psi$ to $H$ and the induced character of $\tilde{\psi}$ to $G$, respectively.

Glasgow Math. J. 22 (1981) 151-154. 
We write $G^{\prime}$ for the commutator subgroup of $G$. If $S$ is a subset of $G, N_{G}(S)$ denotes the normalizer of $S$ in $G$. We use the notation $O_{p^{\prime}}(G), O_{p}(G)$ and $O_{p^{\prime}, p}(G)$ following custom (cf. [3, p. 397]).

THEOREM. Let $B$ be an arbitrary p-block of $G$ with defect group $D$, and let $N=N_{G}(D)$. Then the following are equivalent.

(1) $D \subseteq N_{B}^{*}$.

(2) When $b$ is a p-block of $N$ with $b^{G}=B$, for each $\psi \in \operatorname{Irr}(b)$ there is some $\chi \in \operatorname{Irr}(B)$ such that $\left.X\right|_{N}=\psi$.

(3) When $b$ is a p-block of $N$ with $b^{G}=B$, for each $\psi \in \operatorname{Irr}(b)$ with height zero there is some $\chi \in \operatorname{Irr}(B)$ such that $\left.\chi\right|_{N}=\psi$.

(4) When $b$ is a p-block of $N$ with $b^{G}=B$, for each $\psi \in \operatorname{Irr}(b)$ with height zero there is some $\chi \in \operatorname{Irr}(B)$ such that $\chi$ has height zero and $\left.\chi\right|_{N}=\psi$.

(5) If $\chi \in \operatorname{Irr}(B)$ then $\left.\chi\right|_{N} \in \operatorname{Irr}(N)$.

(6) If $\chi \in \operatorname{Irr}(B)$ and $\chi$ has height zero then $\left.\chi\right|_{N} \in \operatorname{Irr}(N)$.

(7) If $\phi \in \operatorname{IBr}(B)$ then $\left.\phi\right|_{N} \in \operatorname{IBr}(N)$.

Proof. (7) $\Rightarrow$ (1). Since $D$ is normal in $N$, by [3, Theorem 53.9(ii)], $D \subseteq \operatorname{Ker} \tilde{\phi}$ for all $\tilde{\phi} \in \operatorname{IBr}(N)$. Thus, (7) implies (1).

(1) $\Rightarrow(5)$, (7). Let $H=N_{B}^{*}$ and $V=N_{\mathrm{B}}$. By [1, Propositions (3A) and (3D)], $H$ is $p$-nilpotent and $V=O_{p^{\prime}}(H)$. Let $\phi \in \operatorname{IBr}(B)$. Since $H \subseteq \operatorname{Ker} \phi$, we can consider $\phi \in$ $\operatorname{IBr}(G / H)$, so that $\phi \in \operatorname{IBr}(\bar{B})$ for some $p$-block $\bar{B}$ of $G / H$. By [3, Lemma 64.3(1)], $\bar{B} \subseteq B$. Hence, by [3, Lemma 64.3(2)], $D$ contains a Sylow p-subgroup of $H$. Thus, (1) implies that $D$ is a Sylow $p$-subgroup of $H$, so that $H=V D$. Hence, by the Frattini argument [4, I 7.8 Satz], $G=H N=V N$. This proves (5) since $V \subseteq \operatorname{Ker} \chi$ for all $\chi \in \operatorname{Irr}(B)$. Similarly, we get (7) since $H \subseteq \operatorname{Ker} \phi$ for all $\phi \in \operatorname{IBr}(B)$.

(5) $\Rightarrow(6)$. Trivial.

(6) $\Rightarrow(4)$. Let $b$ be a $p$-block of $N$ with $b^{G}=B$. By [3, Corollary 54.11 and Lemma 57.4], $D$ is a defect group of $b$. Let $\psi \in \operatorname{Irr}(b)$ such that $\psi$ has height zero. We can write $\psi^{G}=\sum_{i} u_{i} \chi_{i}$ where $\chi_{i} \in \operatorname{Irr}(G)$ and $u_{i}$ is a non-negative integer for each $i$. Let $|D|=p^{d}$. If $\chi_{i} \in \operatorname{Irr}(B)$ and $u_{i} \neq 0$, then we have

$$
\begin{aligned}
\nu_{\mathrm{p}}\left(u_{\mathrm{i}} \chi_{\mathrm{i}}(1)\right) \geqslant \nu_{\mathrm{p}}\left(\chi_{\mathrm{i}}(1)\right) \geqslant \nu_{\mathrm{p}}(|G|) & -d \\
& =\nu_{\mathrm{p}}(|G: N|)+\nu_{\mathrm{p}}(|N|)-d=\nu_{\mathrm{p}}(|G: N|)+\nu_{\mathrm{p}}(\psi(1))=\nu_{\mathrm{p}}\left(\psi^{G}(1)\right)
\end{aligned}
$$

since $\psi$ has height zero. Hence it follows from [2, (3A)] that there is some $\chi_{i} \in \operatorname{Irr}(B)$ such that $u_{i} \neq 0$ and $\nu_{p}\left(u_{i} \chi_{i}(1)\right)=\nu_{p}\left(\psi^{G}(1)\right)$. This implies that $\chi_{i} \in \operatorname{Irr}(B)$ has height zero by the above inequality. By Frobenius reciprocity, $\psi$ is a component of $\left.\chi_{i}\right|_{N}$. Thus, (6) implies $\left.\chi_{i}\right|_{N}=\psi$.

(4) $\Rightarrow(3)$. Clear.

$(5) \Rightarrow(2)$. Similar to the proof of $(6) \Rightarrow(4)$.

$(2) \Rightarrow(3)$. Clear.

(3) $\Rightarrow(1)$. By Brauer's first main theorem [3, Theorem 58.3], there is a $p$-block $b$ of $N$ with defect group $D$ such that $b^{G}=B$. Let $R=N_{b}$, so that $R \subseteq O_{p^{\prime}}(N)$ from [1, Proposition (3A)]. Let $\phi \in \operatorname{IBr}(b)$. By [1, Proposition (3D)] and [3, Theorem 53.9(ii)], 
$R D \subseteq \operatorname{Ker} \phi$. Hence we can consider $\phi \in \operatorname{IBr}\left(b^{*}\right)$ for some $p$-block $b^{*}$ of $N /(R D)$. By [3, Lemma 64.3(1)], $b^{*} \subseteq b$. Let $\psi \in \operatorname{Irr}\left(b^{*}\right)$. We can consider $\psi \in \operatorname{Irr}(b)$ with $R D \subseteq \operatorname{Ker} \psi$. Clearly, we may consider $\psi \in \operatorname{Irr}\left(N /\left(R D^{\prime}\right)\right)$. Since $(R D) /\left(R D^{\prime}\right)$ is an abelian normal subgroup of $N /\left(R D^{\prime}\right)$, we get from $[4, \mathrm{~V} 17.10 \mathrm{Satz}]$ that $\psi(1)|| N /(R D) \mid$. This shows that $\psi \in \operatorname{Irr}(b)$ has height zero since $D$ is a defect group of $b$. Hence, by (3) there is some $\chi \in \operatorname{Irr}(B)$ with $\left.\chi\right|_{N}=\psi$. This shows $R D \subseteq \operatorname{Ker} \chi$. Hence $\{\chi \in \operatorname{Irr}(B) \mid R D \subseteq \operatorname{Ker} \chi\} \neq \varnothing$. Let $L=\bigcap\left\{\operatorname{Ker} \chi \mid \chi \in \operatorname{Irr}(B), R D^{\prime} \subseteq \operatorname{Ker} \chi\right\}$, so that $R D^{\prime} \subseteq L \cap N$. Let $\psi_{i} \in \operatorname{Irr}(b)$ with $R D^{\prime} \subseteq \operatorname{Ker} \psi_{i}$. As above, $\psi_{i}$ has height zero. Thus, by (3) there is some $\chi_{i} \in \operatorname{Irr}(B)$ with $\left.\chi_{i}\right|_{N}=\psi_{i}$, so that $R D^{\prime} \subseteq \operatorname{Ker} \chi_{i}$. This implies $L \subseteq \bigcap_{i} \operatorname{Ker} \chi_{i}$, so that

$$
L \cap N \subseteq \cap\left\{\operatorname{Ker} \psi \mid \psi \in \operatorname{Irr}(b), R D^{\prime} \subseteq \operatorname{Ker} \psi\right\} .
$$

Next, we want to claim

$$
\bigcap\left\{\operatorname{Ker} \psi \mid \psi \in \operatorname{Irr}(b), R D^{\prime} \subseteq \operatorname{Ker} \psi\right\}=R D^{\prime} .
$$

Let $I=\left\{\psi \in \operatorname{Irr}(b) \mid R D^{\prime} \subseteq \operatorname{Ker} \psi\right\}=\left\{\psi_{1}, \ldots, \psi_{m}\right\}$. We have already shown $I \neq \varnothing$. Take any $\psi_{i}$ with $1 \leqslant i \leqslant m$. Since $\mathrm{RD}^{\prime} \subseteq \operatorname{Ker} \psi_{i}$, we can consider $\psi_{i} \in \operatorname{Irr}\left(\bar{b}_{i}\right)$ for some $p$-block $\bar{b}_{i}$ of $N /\left(R D^{\prime}\right)$. By [3, Lemma 64.3(1)], $\bar{b}_{i} \subseteq b$ for all $i=1, \ldots, m$. Then, $I=\bigcup_{i=1}^{m} \operatorname{Irr}\left(\bar{b}_{i}\right)$. Take any $\bar{b}_{i}$ with $1 \leqslant i \leqslant m$. By [3, Lemma $\left.64.3(1)\right]$, there is a p-block $\tilde{b_{i}}$ of $N / R$ with $\bar{b}_{i} \subseteq \tilde{b}_{i} \subseteq b$. Let $\tilde{F}=\bigcup_{i=1}^{m} \operatorname{IBr}\left(\tilde{b}_{i}\right)$ and $\bar{F}=\bigcup_{i=1}^{m} \operatorname{IBr}\left(\bar{b}_{i}\right)$. Take any $\tilde{b}_{i}$. Let $\phi \in \operatorname{IBr}\left(\tilde{b}_{i}\right)$. Since $\left(R D^{\prime}\right) / R$ is a normal $p$-subgroup of $N / R,\left(R D^{\prime}\right) / R \subseteq \operatorname{Ker} \phi$ from [3, Theorem 53.9(ii)]. Thus, $R D^{\prime} \subseteq \operatorname{Ker} \phi$ if we consider $\phi \in \operatorname{IBr}(b)$. Then, $\phi \in \operatorname{IBr}(\bar{b})$ for some $p$-block $\bar{b}$ of $N /\left(R D^{\prime}\right)$. By [3, Lemma 64.3(1)], $\bar{b} \subseteq b$. Take any $\psi \in \operatorname{Irr}(\bar{b})$, so that $\psi \in \operatorname{Irr}(b)$ with $R D^{\prime} \subseteq \operatorname{Ker} \psi$. This shows $\psi \in I$. Hence $\bar{b}=\bar{b}_{j}$ for some $j$. So that $\phi \in \operatorname{IBr}\left(\bar{b}_{j}\right)$. Thus, we have $\tilde{F}=\bar{F}$. Then,

$$
\begin{array}{r}
\bigcap_{i=1}^{m} N_{b_{i}}^{*}=\bigcap_{\phi \in \bar{F}} \operatorname{Ker} \phi \cong \bigcap_{\phi \in \bar{F}}\left(\operatorname{Ker} \phi /\left(\left(R D^{\prime}\right) / R\right)\right) \\
=\left(\bigcap_{\phi \in \bar{F}} \operatorname{Ker} \phi\right) /\left(\left(R D^{\prime}\right) / R\right)=\left(\bigcap_{i=1}^{m} N_{b_{i}}^{*}\right) /\left(\left(R D^{\prime} / R\right) .\right.
\end{array}
$$

Take any $\tilde{b}_{i}$. Let $\tilde{\psi} \in \operatorname{Irr}\left(\tilde{b}_{i}\right)$. By [1, Proposition $\left.\left(3 \mathrm{~B}^{*}\right)\right], N_{\tilde{b}_{\mathrm{t}}}=O_{\mathrm{p}^{\prime}}(\operatorname{Ker} \tilde{\psi})$. When we consider $\tilde{\psi} \in \operatorname{Irr}(b)$, we write $\psi$ for $\tilde{\psi}$. Then, similarly $R=N_{b}=O_{p}(\operatorname{Ker} \psi)$. Since $\operatorname{Ker} \tilde{\psi}=(\operatorname{Ker} \psi) / R$, $N_{b_{i}}=1$. Hence, by [1, Proposition (3D)], $N_{b_{i}}^{*}$ is a $p$-group for all $i$. Thus, $\bigcap_{i=1}^{m} N_{b_{i}}^{*}$ is also a $p$-group from $\left({ }^{* *}\right)$. Hence, by [1, Propositions (3A) and (3D)], $\bigcap_{i=1}^{m} N_{b_{i}}=1$. Since $\bigcap_{i=1}^{m} N_{b_{i}}=\left(\bigcap_{\psi \in I} \operatorname{Ker} \psi\right) /\left(R D^{\prime}\right)$, we get $\left({ }^{*}\right)$.

Hence, $L \cap N=R D^{\prime}$. Let $K=\bigcap\{\operatorname{Ker} \chi \mid \chi \in \operatorname{Irr}(B), R D \subseteq \operatorname{Ker} \chi\}$. We get $K \cap N=$ $R D$ as for $L \cap N$. Since $D^{\prime} \subseteq L \cap D,(L D) / L$ is isomorphic to a factor group of $D / D^{\prime}$, so that $(L D) / L$ is abelian. We have shown that there is some $\chi \in \operatorname{Irr}(B)$ with $R D \subseteq \operatorname{Ker} \chi$. Hence $K \subseteq \operatorname{Ker} \chi$. We can consider $\chi \in \operatorname{Irr}(\bar{B})$ for some $p$-block $\bar{B}$ of $G / K$, so that $\bar{B} \subseteq B$ by [3, Lemma 64.3(1)]. Then, by [3, Lemma 64.3(2)], $D$ is a Sylow $p$-subgroup of $K$ since $D \subseteq K$. Hence $(L D) / L$ is an abelian Sylow $p$-subgroup of $K / L$. Let $M / L=N_{K / L}((L D) / L)$. By Sylow's theorem, $M=L(M \cap N)$. Hence $M \subseteq L(K \cap N)=L(R D)=L D$, so that $(L D) / L=M / L$. Thus, by Burnside's theorem [3, Theorem 18.7], $K / L$ is $p$-nilpotent. Let $C / L=O_{p^{\prime}}(K / L)$, so that $C \cap L D=L$. Hence, $C \cap D \subseteq L \cap D=L \cap N \cap D=R D^{\prime} \cap D=$ $D^{\prime}$. Since $D$ is a $p$-group, $D^{\prime} \subseteq \Phi(D)$ where $\Phi(D)$ is the Frattini subgroup of $D$. Then it 
follows from [4, IV 4.7 Satz] that $C$ is $p$-nilpotent. Hence, $C=X(C \cap D)$ where $X=O_{p^{\prime}}(C)$. Since $X$ is normal in $K$ and since $K=X D, K$ is also $p$-nilpotent. We know from [1, Proposition (3A)] that $N_{B} \subseteq O_{p^{\prime}}(K)$. On the other hand, there exists $\chi \in \operatorname{Irr}(B)$ with $R D \subseteq \operatorname{Ker} \chi$. By [1, Proposition $\left.\left(3 \mathrm{~B}^{*}\right)\right], N_{B}=O_{\mathrm{p}^{\prime}}(\operatorname{Ker} \chi)$. Since $O_{p^{\prime}}(K)$ is normal in $\operatorname{Ker} \chi, O_{\mathrm{p}^{\prime}}(K) \subseteq O_{p^{\prime}}(\operatorname{Ker} \chi)$. Hence $N_{B}=O_{\mathrm{p}^{\prime}}(K)$. Then, by [1, Proposition (3D)], we have $K / N_{\mathrm{B}} \subseteq O_{\mathrm{p}}\left(G / N_{\mathrm{B}}\right)=N_{\mathrm{B}}^{*} / N_{\mathrm{B}}$. Hence $D \subseteq N_{B}^{*}$. This completes the proof of the theorem.

Corollary 1. Let $G, B, D$ and $N$ be as above and satisfy $D \subseteq N_{B}^{*}$. Let $b$ be a p-block of $N$ with $b^{G}=B$. Then

(i) $p \nmid|G: N|$.

(ii) $k(b) \leqslant k(B)$.

(iii) $k_{0}(b) \leqslant k_{0}(B)$.

Proof. Since there exists $\psi \in \operatorname{Irr}(b)$ with height zero, we get (i) by Theorem (4). We have (ii) and (iii) from Theorem (2) and Theorem (4), respectively.

Corollary 2 (cf. [5, Theorems 2 and 4]). Let $P$ be a Sylow p-subgroup of $G$, and let $N_{0}=N_{G}(P), B_{0}=B_{0}(G)$ and $b_{0}=B_{0}\left(N_{0}\right)$. Then the following are equivalent.

(1) $G$ is p-solvable of p-length 1.

(2) For each $\psi \in \operatorname{Irr}\left(b_{0}\right)$ there is some $\chi \in \operatorname{Irr}\left(B_{0}\right)$ such that $\left.\chi\right|_{N_{0}}=\psi$.

(3) For each $\psi \in \operatorname{Irr}\left(b_{0}\right)$ with $p \nmid \psi(1)$ there is some $\chi \in \operatorname{Irr}\left(B_{0}\right)$ such that $\left.\chi\right|_{N_{0}}=\psi$.

(4) If $\chi \in \operatorname{Irr}\left(B_{0}\right)$ then $\left.\chi\right|_{N_{0}} \in \operatorname{Irr}\left(N_{0}\right)$.

(5) If $\chi \in \operatorname{Irr}\left(B_{0}\right)$ with $p \nmid \chi \chi(1)$ then $\left.\chi\right|_{N_{0}} \in \operatorname{Irr}\left(N_{0}\right)$.

(6) If $\phi \in \operatorname{IBr}\left(B_{0}\right)$ then $\left.\phi\right|_{N_{0}} \in \operatorname{IBr}\left(N_{0}\right)$.

Proof. Clearly, $G$ is $p$-solvable of $p$-length 1 if and only if $P \subseteq O_{p^{\prime}, p}(G)$. By [3, Theorem 65.2(2)], $N_{\mathrm{B}_{0}}^{*}=O_{p^{\prime}, p}(G)$. Since $p \nmid\left|G: N_{0}\right|$, by Brauer's third main theorem [3, Theorem 65.4], the corollary is a special case of the theorem.

REMARK. Concerning kernels of representations of finite groups there is a result of G. O. Michler [6].

\section{REFERENCES}

1. R. Brauer, Some applications of the theory of blocks of characters of finite groups IV, $J$. Algebra 17 (1971), 489-521.

2. R. Brauer, On blocks and sections in finite groups I, Amer. J. Math. 89 (1967), 1115-1136.

3. L. Dornhoff, Group representation theory, parts A and B, (Dekker, 1971-1972).

4. B. Huppert, Endliche Gruppen I, (Springer-Verlag, 1967).

5. I. M. Isaacs and S. D. Smith, A note on groups of p-length 1, J. Algebra 38 (1976), 531-535. 47-49.

6. G. O. Michler, The kernel of a block of a group algebra, Proc. Amer. Math. Soc. 37 (1973),

\section{Department of Mathematics}

ChIBA UNIVERSITY

$1-33$, YAYOI-CHO

CHIBA-CTTY, 280

JAPAN 